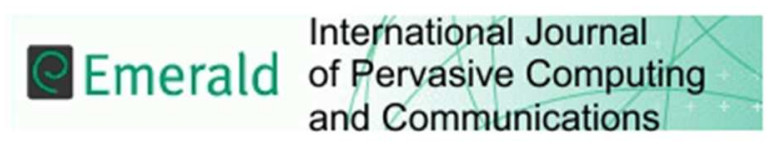

TDAS: A Touch Dynamics based Multi-Factor Authentication Solution for Mobile Devices

\begin{tabular}{|r|l|}
\hline Journal: & International Journal of Pervasive Computing and Communications \\
\hline Manuscript ID & IJPCC-01-2016-0005 \\
\hline Manuscript Type: & Research Paper \\
\hline Keywords: & $\begin{array}{l}\text { Mobile Authentication, Touch Dynamics, Biometrics, Keystroke Dynamics, } \\
\text { Benchmark Dataset }\end{array}$ \\
\hline \multicolumn{2}{|l}{} \\
\hline
\end{tabular}

SCHOLARONE ${ }^{m}$

Manuscripts 


\title{
TDAS: A Touch Dynamics based Multi-Factor Authentication Solution for Mobile Devices
}

\begin{abstract}

\section{Purpose}

The use of mobile devices in handling our daily activities that involve the storage or access of sensitive data (e.g. on-line banking, paperless prescription services, etc.) is becoming very common. These mobile electronic services typically use a knowledge-based authentication method to authenticate a user (claimed identity). However, this authentication method is vulnerable to several security attacks. To counter the attacks and to make the authentication process more secure, this paper investigates the use of touch dynamics biometrics in conjunction with a PIN-based authentication method, and demonstrates its benefits in terms of strengthening the security of authentication services for mobile device.
\end{abstract}

\section{Design/methodology/approach}

The investigation has made use of three light-weighted matching functions and a comprehensive reference dataset collected from 150 subjects.

\section{Findings}

The investigative results show that, with this multi-factor authentication approach, even when the PIN is exposed, as much as 9 out of 10 impersonation attempts can be successfully identified. It has also been discovered that the accuracy performance can be increased by combining different feature data types and by increasing the input string length.

\section{Originality/value}

The novel contributions of this paper are two-fold. Firstly, it describes how a comprehensive experiment is set up to collect touch dynamics biometrics data, and the set of collected data is being made publically available, which may facilitate further research in the problem domain. Secondly, the paper demonstrates how the dataset may be used to strengthen the protection of resources that are accessible via mobile devices.

\section{Keywords}

Mobile Authentication, Touch Dynamics, Biometrics, Keystroke Dynamics, Benchmark Dataset.

\section{INTRODUCTION}

Mobile devices have become an integral part of our routine activities. This is particularly the case with the rapid growth and widespread use of smartphones and digital tablets. The processing capability of these devices has advanced up to the point that most digital activities that can be accomplished on workstations or laptops can also be performed on these portable devices. Routine activities, such as personal and corporate e-mail communications, on-line banking transactions, accessing paperless prescriptions services, route navigation, etc. can also be carried out ubiquitously with these devices.

According to a forecast by networking giant Cisco (Cisco, 2015), there will be approximately 11.5 billion mobile-connected devices by 2019, and the global mobile data traffic will increase nearly tenfold between 2014 and 2019, reaching 24.3 exabytes per month by 2019 . This shows our increasing reliance on mobile devices, and also implies that our private and sensitive date will increasingly be handled, managed and processed by these devices. Therefore, the security of accessing mobile devices and accessing data, services and other resources through the mobile devices is of a prime concern. More stringent security services (or measures) should be embedded in mobile devices. One of these services is user authentication, i.e. how to securely verify a claimed identity.

Authentication is the first-line of defence in any computer system or device as it is a pre-requisite for several other security services such as authorisation and accountability. In a mobile device context, authentication is typically achieved via a knowledge-based authentication method, and with this method, a user proves their identity by demonstrating the knowledge of a secret. This secret could be a PIN (Personal Identification Number), a password, a shared secret (which is similar to a password, but with higher entropy) or a private key corresponding to a public key certified in a digital certificate. Authentication by using a low entropy secret (such as a PIN or a password) is vulnerable to a number of security attacks, e.g. theft of a mobile device, shoulder spoofing and brute force attacks. Authentication by using a high entropy secret (using a shared secret or a private key) usually requires some means to store the secret securely and this may hinder usability and/or introduce a higher cost. To address these issues and to make the authentication service more secure, while, at the same time, without hindering usability, we have been working on integrating a biometric-based (touch dynamics) authentication method with a knowledge-based (PIN) authentication method, called a Touch Dynamic based multi-factor Authentication Solution (TDAS).

This paper reports our effort on the creation and use of a touch dynamics dataset to investigate the benefits of integrating touch dynamics with a PIN-based authentication method. It describes the process of collecting a comprehensive reference dataset consisted of two sets of input PINs from 150 subjects, the extractions of feature data from the dataset, and the use of the extracted feature data as a second authentication factor. The extracted feature data are timing, finger touch size and pressure feature data. The data are classified by using three light-weight classification algorithms, and are used to support the identification of a user in addition to using the PIN-based authentication method. The experiments show that this two-factor approach to authentication can make impersonation attacks much more 
difficult, significantly increasing the assurance level of the authentication process. The paper has also discussed various factors that may impact the accuracy performance of TDAS, such as the timing resolution of feature data type, combinations of feature data types, subject size and input string length.

The structure of this paper is as follows. The next section gives an overview of touch dynamics biometrics. Section 3 explains the experimental setup and the methods and procedures used in collecting (i.e. acquiring) the touch dynamic biometrics data. Section 4 describes the properties of the dataset. Section 5 describes potential feature data that can be extracted from the dataset and, for proof-ofconcept, it illustrates how the captured feature data may be used for authenticating users in a mobile context and the level of improvements in terms of accuracy performance (Section 6). Section 7 critically analyses the related work in the problem context, compares our dataset with other public datasets and outlines open issues and potential research directions. Finally, Section 8 concludes the paper.

\section{BACKGROUND}

\subsection{Overview of Touch Dynamics}

Prior to the emergence of touch dynamics, one of the earliest research work on using keystroke dynamics (i.e. the patterns of interactions between human input and a physical keyboard) to identify users was by (Gaines et al., 1980). They attempted to recognise 6 professional secretaries by analysing the way they typed three passages of text consisting 300 to 400 words each. Since then, keystroke dynamics either on workstations (Bleha et al., 1990; Obaidat, 1995) or in a web-based environment (Cho et al., 2000; Stewart et al., 2011) have been the major topic of research. With the rapid development of mobile communication technologies, recent research efforts in the area have been focusing on mobile devices with physical keypads (Campisi et al., 2009; Clarke and Furnell, 2007). According to the literature survey of related works in the topic area of keystroke dynamics (Teh et al., 2013), since 2007, there has been growing efforts on examining the possibility of applying the concept of keystroke dynamics to user authentication on mobile platforms. More recently, the research activities are largely carried out in the context of touchscreen mobile devices (Saravanan et al., 2014; Dhage et al., 2015).

Touch dynamics refers to the process of measuring and assessing human touch rhythm on mobile devices, such as digital tablets, smartphones, or touchscreen panels. When a human interacts with a mobile device, a digital signature is generated. The signatures generated by different individuals are believed to be rich in discriminative properties, which hold potentials as personal identifiers. The availability of higher resolution sensors in recently released mobile devices provide added opportunities to the development of touch dynamics biometrics, as these sensors allow the extraction of more discriminative feature data types. Touch dynamics biometrics can be integrated with an existing knowledge-based authentication method to form a so-called multi-factor authentication solution. Such a solution can make unauthorised accesses to mobile devices harder, thus strengthening the security level of mobile devices. Using touch dynamics to identify a user has its unique advantages, and, at the same time, it also introduces challenging issues. The following highlights the advantages and challenging issues.

\subsection{Advantages}

Transparency. A touch dynamics based authentication method requires little or no additional interventions from a device user. This is because the capture and processing of touch patterns can be carried out in the background while the user is using the device. Users may not even be aware that they are being authenticated periodically or are being protected by an extra layer of authentication. This is in a stark contrast to other biometrics based authentication methods that usually requires explicit alignment of a biometrics feature to a specific sensor. For example, in the case of iris recognition, a user is required to look straight into an intra-red camera to take an iris image, and in the case of finger based authentication, a user needs to put one of his/her fingers on the fingerprint sensor.

Familiarity. The touch dynamics feature data used for authentication is collected during mobile users' routine input activities. This is a process which mobile users are already familiar with, so the feature data collected tend to have a gentler learning curve with a higher usability level.

Revocability. The touch dynamics feature data can be replaced should a passcode associated to a touch dynamics pattern is compromised, as a new touch dynamics template can easily be associated to a new passcode. However, this is not the case for other physiological biometrics, e.g. for iris or face biometrics, once they are compromised, there will be no replacement, and for fingerprints, the number of replacements are limited (there are only 10 fingers to use after all).

Non-dependency. The feature acquisition of touch dynamics is less sensitive to environmental factors. Therefore, it is more suited to, and can be more easily deployed in, a mobile context. A mobile device is usually operated in an on-the-go manner, so the values of some environmental factors, such as the screen lighting level and background noise level, are constantly changing. Other biometrics features such as iris or voice biometrics are sensitive to these environmental factors.

Cost Effectiveness. In contrast to other physiological biometrics systems such as iris and fingerprint recognitions, which typically require the use of some specialist hardware, touch dynamics recognition only requires the use of build-in mobile sensors. This can reduce device costs and it is ideal for large-scale deployments.

\subsection{Challenging Issues}

Algorithm and Communication Costs. Computational capabilities of mobile devices are typically lower than desktop computers. This means that certain criteria such as algorithm complexity, communication cost and authentication delay are important and should be considered in the design of touch dynamics based authentication solutions. In other words, computational and communication costs introduced as the result of deploying this authentication means should be minimal.

Energy Consumption. Mobile devices, unlike their desktop counterparts, are typically battery powered, so the less the energy an application consumes, the longer the device can operate. Though communication is the major consumer of the power of a device, the number and 
usage frequencies of various sensors embedded in a mobile device, which are used to extract touch feature data, also have a direct impact on the mobile device battery consumption level. Various measures, such as reducing the sampling rate or frequency of data sensing (Niu and Chen, 2012), or performing complex computations only when a device is being recharged (Crawford et al., 2013), have been proposed to reduce power consumption of a mobile device.

Non-Permanence. Usually, human behavioural characteristics change more frequently than physiological characteristics. Similarly, a user's touch dynamics may change gradually as the user is getting more familiar with the passcode, input method, device, and other external factors. Adaptive approaches (Xu et al., 2014) have been proposed in literature to take into account of any gradual changes in touch patterns.

\subsection{Operational Process}

Figure 1 shows a typical touch dynamics biometrics authentication system. From the figure, we can see that the operation of this system can largely be captured in two major phases: (i) User Enrolment, where touch pattern feature data are collected, processed and stored as a reference template, and (ii) User Authentication, where a test sample is compared against the stored reference template(s) to determine the similarity. The two operational phases are accomplished by a number of functional blocks (i.e. architectural components), each of which performs a well-defined function or operation. These components and their respective operations are described below.

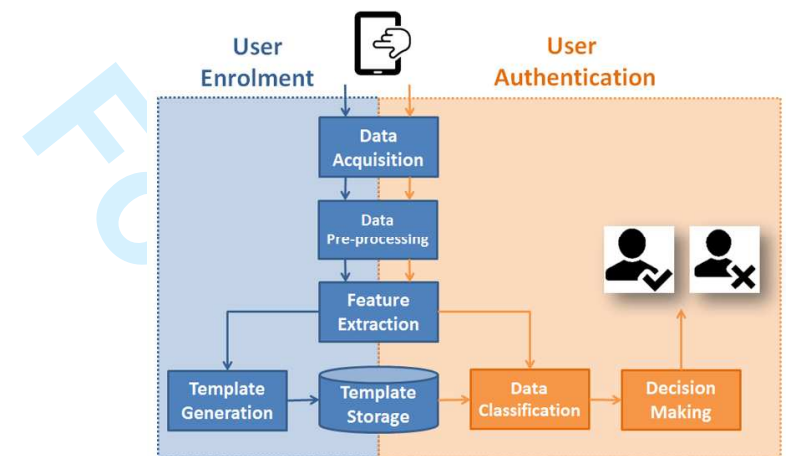

Figure 1. Touch dynamics biometrics authentication framework

Data Acquisition. Data acquisition is an operation by which raw touch feature data are collected. This is usually carried out as the first step and during the setup stage of a touch dynamics biometrics authentication system. The collected raw feature data typically consist of repetitive input samples or collections of input samples over a specified period of time. Devices commonly used in the data acquisition experiments are commercial off-the-shelf smartphones (Buschek et al., 2015) or in some occasions digital tablets (Saravanan et al., 2014).

Data Pre-processing. Once the raw feature data are collected, a data pre-processing operation is carried out to remove outliers in the raw feature data, improving data quality and accuracy performance. Additionally, to optimise computational efficiency on resource limited mobile devices, a dimension reduction technique may also be used to ensure that the selected raw feature data remain small yet representable (de Mendizabal-Vazquez et al., 2014).

Feature Extraction. Feature extraction is another mandatory operation which is carried out in both the enrolment and authentication phases. The main task of this operation is to identify and extract distinctive features common to a subject from the collected raw data. These features will later be used for template generations.

Template Generation. Depending on the classification algorithm selected, the template generation can be an operation that is used to transform the extracted touch feature into a compact form or an operation that trains, selects and stores classification models that uniquely represent each user's touch dynamics characteristics.

Data Classification. This is the major operation for most biometrics systems, where feature data are categorised and compared against reference templates or models. The outcome of this operation is normally associated with a matching score used for decision-making.

Decision Making. This is the last operation carried out by a touch dynamics authentication system in authenticating a user. It is to determine if a presented touch pattern is indeed originated from the target subject. The decision is usually made by comparing the similarity or dissimilarity score generated from a classifier against a predefined threshold (Bo et al., 2014).

\section{EXPERIMENT SETUP}

This section describes and justifies the experiment setup in which raw touch feature data are collected.

\subsection{Deployment and Working Modes}

A touch dynamics biometrics system may be deployed in one of the two modes, identification and verification. Each of these modes functions uniquely and serves a different purpose and use case scenario. The purpose served by the identification mode is to classify unknown touch dynamics samples. This mode is typically deployed for forensic investigations and intrusion detections. Its use on mobile devices is rather limited. The verification mode, on the other hand, is typically used to prove or verify a claimed identity.

The verification mode of touch dynamics biometrics can be further divided into two working modes: a static and a dynamic working mode. In the static working mode, a user is authenticated at the initial instance of, or at some pre-defined intervals during, the user-to-system interaction. Unlike the case for the static working mode, in the dynamic working mode, a user may be authenticated at any instant of a user- 
to-system interaction or for every service access (i.e. continuously) throughout a service access session (in addition to the initial authentication). The functions performed in both modes are complimentary, which means that they can be deployed alongside each other to enhance the security of mobile devices or the security of service access using mobile devices. Our experiment is conducted under the assumption that the static working mode of the verification mode is used.

\subsection{Data Collection Devices}

As mentioned above, there are different types of mobile devices, e.g. featured phones, smartphones, digital tablets and laptops. Most research works in relation to touch dynamics are carried out using various mobile phones. In comparison with mobile phones, digital tablets have a relatively larger screen resolution, which means that a higher subject input variation, and therefore a better feature discrimination, can be captured (Saravanan et al., 2014). For this reason, we have chosen to use a digital tablet as our data collection device. The device is a commercial off-the-shelf Samsung Galaxy Tab 10.1 (GT-P7510) digital tablet. It has a 10.1" widescreen, and is powered by 1GHz dualcore processor and equipped with a 1-GB RAM. The device runs under Android 4.0.4 (Ice Cream Sandwich) and a data collection tool that was developed using Java and Android API Level 15. The entire data collection process was performed using this tablet. The justification for using a predefined device, rather than the devices chosen by the subjects, is to remove any uncontrolled variables such as subject preferences, program compatibility and functionality differences. In this way, the results obtained from the experiment can better capture the discriminative power of touch dynamics feature data and the classification algorithm used.

\subsection{Data Collection Environments}

There are three main environments in which raw touch feature data may be collected: (i) while the subjects carry out their activities as usual; (ii) under a controlled laboratory environment in a fixed location; or (iii) in multiple fixed locations. The first option is expensive, as due to the ubiquitous nature of mobile devices, subjects are likely to be on-the-move and following the subjects while collecting the data may not be convenient and could be costly. The second option is least costly in terms of setting up and running the experiments, but the data collected may not give a true reflection of real world scenarios. To balance costs/feasibility with real-life situations, we have chosen to use the third option, i.e. we let subjects to choose their preferred locations where their touch dynamics are extracted. The locations used included offices, homes, inside cars, classrooms, cafés, and public areas.

\subsection{Collection Method}

Data should be collected when subjects are in a stable state, i.e. after they are familiar with the device input facility and the data collection procedure, as, otherwise, data collected may not properly capture subjects' input features. Data captured improperly may increase false acceptance and false rejection rates when they are used to authenticate the subjects. According to (Ngugi, Tremaine, et al., 2011), input patterns, styles or speeds, can vary and stabilise over time. To ensure data are collected after the patterns, styles and speeds, are stabilised, and to reduce the effect of subjects' unfamiliarity with the input facility and procedure, one of the two approaches may be used. The first one is to divide a data collection session into multiple sub-sessions and the sub-sessions are separated by a selected time frame. For example, we may have 4 sub-sessions each with 1 week apart, and the data collected in multiple sub-sessions are cumulatively merged into a single set. This approach provides a good level of accuracy, but may suffer from a high dropout rate (De Luca et al., 2012), as the data collection process is usually carried out on a voluntary basis and we cannot expect all the participants to take part in all the sub-sessions. As a result, the sample size of the dataset may be reduced. The second approach is to collect data in a single session, but the subjects are asked to familiarise with the input facility and procedure as many times as necessary before a data collection process actually takes place. The entire data collection process takes an average of 15 to 20 minutes (in addition to the time taken to familiarise the input device and procedure). This latter approach is commonly used in experiments reported in the literature (Chong et al., 2010; Kim et al., 2010; Loy et al., 2007). So in our experiments, we have chosen to use the second approach.

\section{DATASET}

\subsection{Subject Size}

The subject size refers to the number of subjects from whom data are collected. Typically a subject size of greater than 100 subjects is regarded as a large subject size (Teh et al., 2013). Using a larger subject size can provide more data to verify the scalability of a chosen classifier as mentioned in (Clarke and Furnell, 2007). Most of the relevant works in the touch dynamics domain were carried out with a subject size smaller than this value. Only a handful published works (Gascon et al., 2014; Serwadda et al., 2013; Trojahn et al., 2013) use a subject size greater than 100 subjects. However, in the latter group of works, the subjects involved were restricted to a certain profession and the datasets were not made publically available for evaluation. At the time of this writing, our dataset contains touch dynamics data of 150 subjects with a diverse range of professions. The dataset is grouped in 3 different packages. The first package contains data from 50 subjects, the second package contains data from 100 subjects, and the third package contains data from all of the 150 subjects. In this way, the datasets may be used, in different ways, for comparisons between different subject sizes within the same subject grouping.

\subsection{Subject Demography}

Experimental subjects are typically selected based on some criteria. The commonly used criteria are age distribution, population mixture, and profession diversity. Subjects from different age groups, with different backgrounds and/or different professions tend to use their devices at different frequencies. Therefore, if the subjects are not selected properly, there may be unintended bias in the experimental results. To reflect real world situations as much as possible, the demography of the subjects taking part in the data collection should be as diverse as possible. In other words, people from different age groups, of different genders and with different device usage frequencies should be represented as much as possible.

Most of the published works are based on subjects that are selected from: (i) a narrow age distribution (i.e. 19-26) (Antal and Szabó, 2014), (ii) confined to only people within the same organisation (i.e. within research institute) (Giuffrida et al., 2014), or (iii) restricted to limited profession (i.e. students) (Draffin et al., 2014). These options are frequently selected because they are less costly and may be easier to 
conduct the experiments. Data collected in this way may not properly capture the biometric features from a wider community. (El-Abed et al., 2014; Kolly et al., 2012; Trojahn et al., 2013) are the few pieces of work we are able to find in the literature, which recruited subjects from dissimilar age groups, and diverse population groups and professions. Inspired by these works we have made our best effort to reach out to the general public within our resource budget. Table 1 summarised the demography of the subjects recruited in our experiment.

Table 1. Subject demography of our dataset

\subsection{Input Type}

Input string types are an important experimental variable that should be considered in touch dynamics biometrics research. This is because the feature used for touch dynamics biometrics is extracted from a subject's input string. Generally, experimental subjects are required to provide character-based, numerical-based (PIN) or other non-specific touch events. PIN input has been the most widely used authentication method for mobile devices, so we first used a 4-digit numerical input ("5560"), and then a 16-digit numerical input ("1379666624680852"). The use of two different PIN lengths allows us to evaluate the effects of different input string lengths experimentally. These two predefined numbers were carefully chosen with the following key positioning combination strategies:

- Apart: keys are separated by at least one key apart.

- Repetition: reoccurrence of identical key.

- Adjacent: keys located diagonally to each other.

- Sequence: keys situated horizontally or vertically to each other.

These positioning strategies were used to spread the variety of input strings. A graphical illustration of the approach is depicted in Figure 2. Requiring all the subjects to use a single predefined input string (for each string length) allows us to increase the number of impersonation samples available for our testing phase (Section 6.2) without the need for collecting additional data.

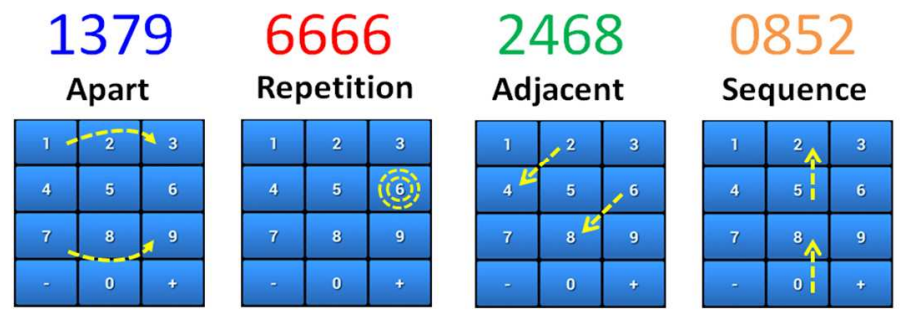

Figure 2. Four different key positioning strategies

\subsection{Sample Size}

The input sample size affects the accuracy, robustness and outcome of an experiment (Sen and Muralidharan, 2014). A larger number of samples used would allow us to gain a better representation of a subject's touch dynamics behaviour, and, as a result, achieve a higher level of accuracy (Tasia et al., 2014). Though it is desirable to have a large number of sample data, it is not always practical to expect subjects to repeat the input for a large number of times during the enrolment stage. This is because subjects may not be available for a long stretch of time, or may feel uncomfortable with a lengthy acquisition procedure (Tasia et al., 2014). Therefore, selecting an optimal input sample size for each data acquisition session is necessary.

According to the work reported in literature, the benchmark for the number of samples collected per subject per session is somewhere between 10 to 20 repetitions for a fixed input type (Buschek et al., 2015; Sen and Muralidharan, 2014; Trojahn et al., 2013). Therefore, in our data collection process, subjects were required to repeat each input string for 10 consecutive times, resulting in 20 samples per subject ( 10 for the short digit sample and 10 for the long digit sample). In terms of error handling, any input mistake made by a subject was automatically discarded and the subject was prompted to repeat that particular input sample, and this is a common practice as explained in the literature (Campisi et al., 2009; Maiorana et al., 2011; Robinson et al., 1998).

\subsection{Raw Feature Representation}

A number of application programming interfaces (APIs) have been used to capture a subject's feature data. In detail, each single screen touch event (finger touching down or lifting up from the touchscreen) is detected by the onTouchListener API. The timestamps of each key press and release are logged by invoking the nanoTime() API. This API returns the most precise time (in nanoseconds) that is available on the device. We also use the API functions, getSize() and getPressure(), under the MotionEvent class to retrieve the values of finger circumference and pressure, respectively. These functions return a normalised decimal value between 0 and 1 . However, we have noticed that getPressure() always returns a value of 1.0. We have tried to resolve this issue but no success. However, as some other devices also encounter the same problem, we anticipate that this problem will be resolved by the mobile operating system's provider in their subsequent API version update. Each completed touch event on a key generates two timestamps $\left(t_{\text {press }}\right.$ and $\left.t_{\text {release }}\right)$, a finger touch size $(p s)$ and a pressure value $(p v)$. The data collected from these events will go through feature extraction and template generation process. For each repeated input sample $(r)$ of raw touch dynamics data, the feature data and the particular key press $\left(k_{\text {press }}\right)$ and key release $\left(k_{r e l e a s e}\right)$ are recorded using the format shown below and the recorded data are stored in a separate file for each subject.

$$
r, k_{\text {press }}, t_{\text {press }}, k_{\text {release }}, t_{\text {release }}, p s, p v
$$




\section{METHODOLOGY}

\subsection{Feature Extraction}

Two types of features are captured. These are timing data and finger touch size $(p s)$. Both are captured during the subject-to-device interactions with the input keys. $p s$ is obtained directly from the returned value of an Android API function without further customisation. However, for timing data acquisition, some manipulation to the touch event timestamps is required.

A timing data can be extracted in different feature length. The shortest feature length is known as uni-graph, which is the timing data extracted between touch event timestamp values of the same key. Subsequently, the timing data extracted from two or more keys are called di-graph and $\mathrm{n}$-graph, respectively. Figure 3 shows the different $\mathrm{n}$-graph sizes for a given sample input string. In our experiments, we have chosen to extract timing data of uni-graph and di-graph.

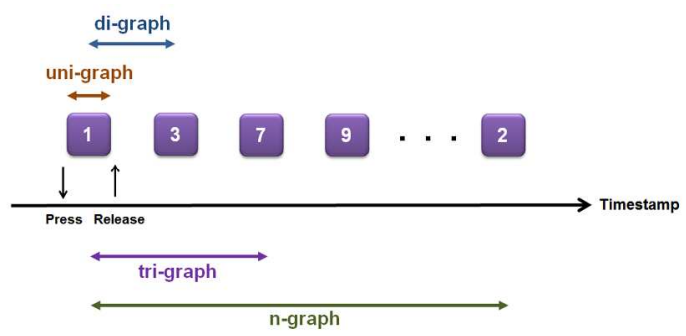

Figure 3. The different timing data feature length

The timing data extracted can be further divided into two categories: (1) Dwell Time (DT), i.e. the time duration for the touch action of the same key (also known as interval, press or hold time); (2) Flight Time (FT), i.e. the time interval between the touch actions on two successive keys (also known as latency). As shown in Figure 4, there are four variants of $F T$.

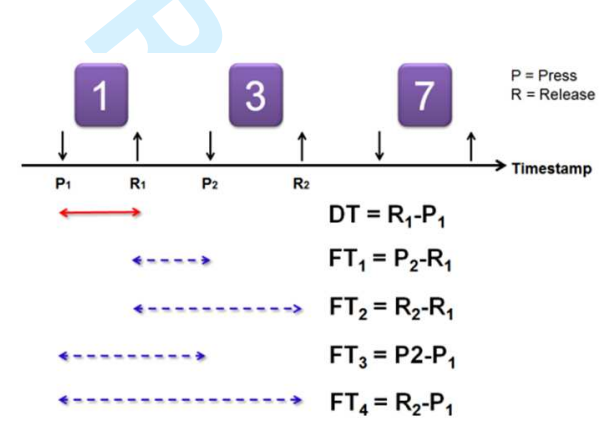

Figure 4. Types of timing feature data extracted

\subsection{Template Generation}

Template generation is a process by which a subject's touch feature samples are combined and transformed into a compact yet representative structure. A subject's template should uniquely capture the subject's touch feature. For each subject, we use six feature data types, and, for each feature data type, a template data is generated. This template data consists of two items, a mean $(\mu)$ value and a standard deviation $(\sigma)$ value. The equations below show how the template data for a feature data type, $D T$, is calculated. For example, given a training sample set of $n$ number of $D T$, the template data for $D T$ is calculated as:

$$
\begin{gathered}
\mu=\frac{1}{n} \times \sum_{i=1}^{n} D T_{i} \\
\sigma=\sqrt{\frac{1}{n} \times\left(\sum_{i=1}^{n} D T_{i}^{2}-\frac{\left(\sum_{i=1}^{n} D T_{i}\right)^{2}}{n}\right)}
\end{gathered}
$$

The same procedure and computation are applied to other feature data types.

\subsection{Classifier}

We use three matching functions to, respectively, compute and compare the likeliness of a test sample against a reference template feature. The likeliness, which is measured in terms of a similarity score $(s)$, is computed by feeding the test sample value $(\tau)$ of a feature vector element of position $(i)$ and the value of $\mu$ and $\sigma$ from the reference template into each function, i.e. $s_{i}=f\left(\tau_{i}, \mu_{i}, \sigma_{i}\right)$. The three matching functions used are Gaussian Estimation (GE), Z-Score (ZS), and Standard Deviation Drift (SD), as given below:

$$
f_{G E}\left(\tau_{i}, \mu_{i}, \sigma_{i}\right)=e^{-\frac{\left(\tau_{i}-\mu_{i}\right)^{2}}{2 \sigma_{i}^{2}}}
$$




\begin{abstract}
where $n$ refers to the total elements in the feature vector considered, and $D_{\text {final }}$ is the final acceptance or rejection decision of a given test
\end{abstract} sample.

\title{
6. PERFORMANCE EVALUATION
}

\subsection{Evaluation Criteria}

To evaluate the accuracy level of a biometrics authentication system, three main metrics are commonly used. These are the False Acceptance Rate (FAR), False Rejection Rate (FRR) and Equal Error Rate (EER). FAR is the percentage ratio of the number of illegitimate trails that are falsely accepted against the total number of illegitimate trials. A lower FAR indicates fewer illegitimate trails are falsely accepted, thus the higher the security level of the biometrics authentication method. FRR is the percentage ratio of the number of legitimate trials that are falsely rejected against the total number of legitimate trials. A lower FRR indicates fewer legitimate trails are falsely rejected, thus the higher the usability of the method. The third performance matric, EER, is a single-number performance metric, which is commonly used to measure and compare the overall accuracy of different biometrics systems. EER is obtained by first plotting a graph for each of FAR and FRR against a matching threshold and then taking the interception point of the two graphs. Typically, the lower the FAR and FRR values, the lower the EER value. A lower EER value indicates a better performance of the biometrics authentication method. However, it is impractical to lower both FAR and FRR simultaneously as FAR and FRR are negatively correlated. Therefore, in practice, we usually choose the threshold value to achieve a required security level.

\subsection{Training and Testing Setup}

For each subject recruited, 2 sets of 10 input samples are collected, one set for the 4-digit input and the other for the 16-digit input. For each of the two input length categories, 7 out of 10 are used for training (i.e. for generating a template for the subject) and the remaining 3 for testing (i.e. for estimating the FAR and FRR values). To reduce intra-session variations, the 7 samples are selected randomly from the 10 -sample set. The samples used for training are not reused for testing so that the performance assessment can be made independent of the model development. In a FAR test, a subject's template was compared against all the other subjects' testing samples. This process was reiterated for all the subjects' templates. As there are a total of 150 subjects recruited and each subject has 3 testing samples, the total number of illegitimate trials conducted is $150 \times(150-1) \times 3=67,050$. In a FRR test, a subject's template is compared against the subject's own testing samples. As there are a total of 150 subjects and each subject has 3 testing samples, the total number of legitimate trials is $150 \times 3=450$.

\subsection{Results Discussion}

\subsubsection{Timing Feature Data Scaling}

The accuracy of timing feature data extracted from timestamps is influenced by the timing resolution used, so it is important to choose an appropriate timing resolution before extracting the timing feature data. As discussed in section 5.1, timing feature data are extracted by subtracting the timestamps of different touch actions (i.e. a key press or release). By default, the timestamps recorded by a mobile device's nanoTime() API has a timing resolution in the order of nanoseconds. This resolution is inappropriate because a human's tapping speed is usually at a much slower pace than this order. To solve this problem, an original timestamp $(t)$ should be normalised by a scaling factor $(s)$ to create a normalised timestamp $\left(T_{\text {norm }}\right)$ with the chosen resolution, and this can be done by using the following formula:

$$
T_{\text {norm }}=t \times e^{-s}
$$

To investigate the effects of using different scaling factor values, we have calculated the EER values of timing feature data extracted from timestamps normalised with different scaling factor values. Figure 5 shows the EER values of the timing feature data type $F T_{4}$. The same trend has been observed for all the other timing feature data types. As can be seen from the figure, the EER values are higher when the scaling factor uses a very small or a very big value; the EER values for both input string lengths decrease when the scaling factor value increases from 1 to approximately 4 , plateau from 4 to 8 , then they start to increase sharply. This result may be explained as follows. When timestamps are normalised by using a smaller or moderate scaling factor, the timing feature data gradually resembles better a typical human tapping speed, and so is the ability to better represent a human's touch pattern. However, when the scaling factor of 9 is used, the 
timestamp value becomes so small that it conveys little useful timing information. Therefore, when an extreme scaling factor value is used, the EER value increases sharply. In this experiment, we have chosen to use a moderate scaling factor value of 5.

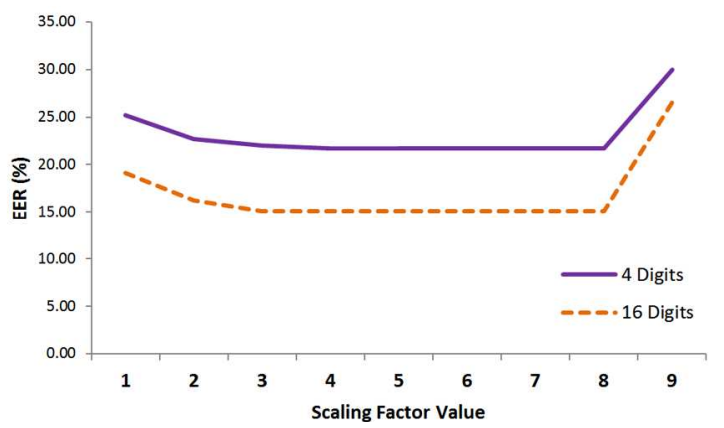

Figure 5. Accuracy performances of different scaling factor values.

\subsubsection{Single Feature Data Types}

Experiments have been conducted to investigate the accuracy performances of different feature data types. As shown in Figure 6, the $p s$ feature outperforms all timing related feature data types for both the 4-digit and 16-digit input strings. This may be due to the fact that ps could capture more properties from a subject's touch pattern. For example, the amount of force used, finger arrangement, touch angle and finger thickness. The mixture of these properties establishes a distinctive pattern, which can better capture the uniqueness in each subject's touch pattern.

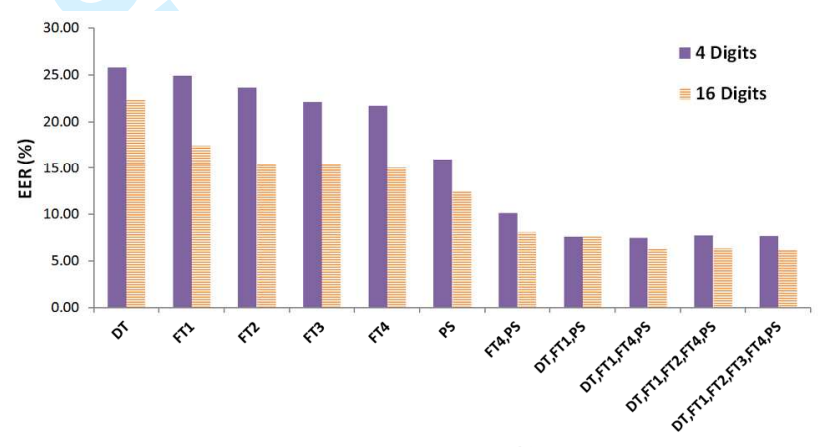

Feature Data Types Combination

Figure 6. Accuracy performances of single vs different feature data type combinations.

As for timing related feature data types, the accuracy performance of any variant of $F T$ is slightly better than $D T$. This implies that the time taken for a subject's finger to traverse from one key to another has more discriminative power than how long a key is hold down. Throughout the data collection process, we observed that the key combinations entered by different subjects are different even if the input strings entered are identical. This may be due to the fact that breaking up an input string into multiple smaller subsets (i.e. chunks) can make it easier to memorise (Ngugi, Kahn, et al., 2011). The information embedded within the natural short pauses between different chunks may have increased the uniqueness of $F T$. This is particularly the case for strings with a longer string length, due to the increased number of possible chunk combinations as shown in Figure 7.

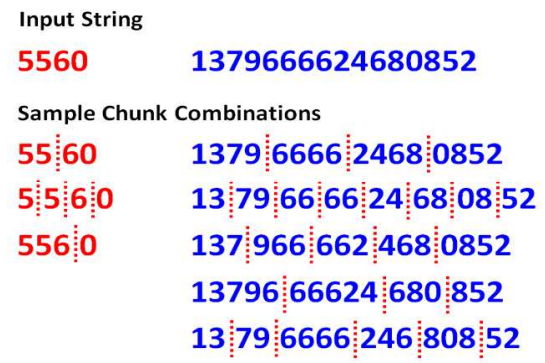

Figure 7. The different possible chunk combinations of different input string.

\subsubsection{Combining Different Feature Data Types}

Although $p s$ proved to be the best feature data type in terms of EER, its EER performance of 15.98\% (the 4-digit string) and $12.44 \%$ (the 16-digit string) are still rather unsatisfactory. To improve this performance, we have combined $p s$ with different combinations of timing feature data types. So, in a given authentication instance, multiple feature data types are used, and the decision made for the authentication instance is made by combining the decisions made on each chosen feature data type using the AND voting rule. Table 2 shows the best EER values of different feature data type combinations. The values in brackets indicate the accuracy performance gains for using different feature data type combinations against single feature data type. As can be seen from the table, the more feature data types used, the lower 
the EER value, the better the accuracy performance. The lowest EER value is achieved when all the six feature data types are used. In this case, the EER values for the 4-digit and 16-digit input strings are, respectively, $7.71 \%$ and $6.27 \%$, which are less than half of the respective EER values when $p s$ is used alone.

\section{Table 2. EER values of different feature data type combinations}

From the results shown in Figure 6, it is clear that combining two or more feature data types can lead to a significantly better accuracy performance than using a single feature data type. This indicates that when more feature data types are used to train the model, the ability to distinguish between a legitimate and an illegitimate user sample also increases.

\subsubsection{Input String Lengths}

Input string lengths may also affect the accuracy performance of a biometrics authentication system. To investigate the effect, we have calculated the EER values using the three matching functions and two sets of PINs with respective string lengths of 4 and 16 digits. The 4 digit set represents a short input string case, while the 16-digit set represents a long input string case. The analysis results are plotted in Figure 8. As can be seen from the figure, a longer input string leads to a lower EER value, which indicates a better accuracy performance. This result can be explained as follows. When the input string length increases, feature data samples within each input string, and the number of different chunk combinations also increase, and so is the ability to better capture a subject's touch pattern. In addition, when the input string length increases, the number of illegitimate feature data samples required to match that of a legitimate reference template will also increase. Therefore, the longer the input strings, the better the accuracy performance one could achieve from a PIN based biometrics authentication system.

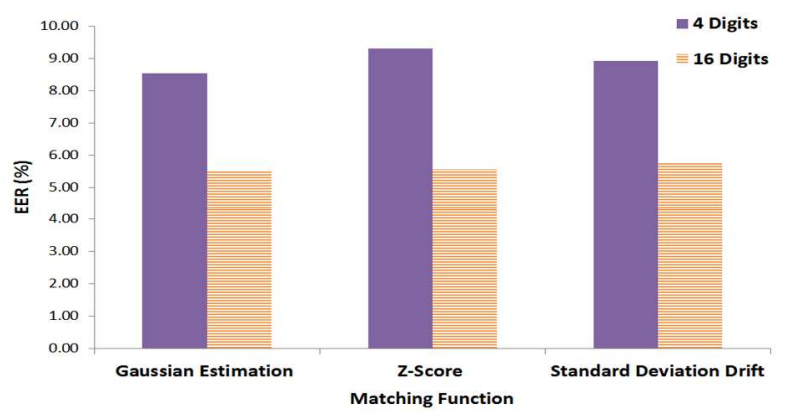

Figure 8. The effects of the input string lengths on the EER values

\subsubsection{Subject Size}

We have also investigated the effects of subject sizes on the EER values. This experiment is based on three matching functions, GE, ZS, and SD. Two subject sets are used: one set has 50 subjects and the other 150 subjects. For each subject set, we used two input string, $4-$ digits and 16-digits. The experimental results are shown in Table 3. Ideally, an input string tested on a larger subject size should achieve a lower or at least a comparable error value than when tested on a smaller subject size. If this case holds, it indicates that the proposed method is scalable at different subject sizes. As shown by the results in the table, when the input string length is 16-digits, the differences in the EER values produced by using different matching functions remains fairly constant when the subject size goes up from 50 to 150 . This indicates that the 16-digits input string can provide us with more consistent experimental results. From the table, we can also observe that when the subject size of 50 is used, the average EER value decreases slightly from 7.93 to 6.81 as the input string length increases from 4digits to 16-digits. However, when the subject size is 150 , this average value decreases considerably from 8.92 to 5.59 as the input string length increases from 4-digits to 16-digits. This inconsistency may be due to the fact that an input string with a shorter length is less able to discriminate different touch patterns (as discussed in the previous section).

\section{Table 3. EER values vs subject sizes}

\subsubsection{Classifier Performance}

The EER values produced by all the three functions are shown in Table 4. Two input string lengths, i.e. 4-digits and 16-digits, are used and the subject size chosen is 150 . From the results, it can be seen that, generally, the fluctuations in the results produced by the different classifiers are smaller when the input string length is 16 digits in comparison with the 4 digits input string length. For example, when the input string length is 16 -digits, the differences in the EER values are less than $0.25 \%$, whereas when the input string length is 4 digits, the differences are at least $0.37 \%$. Among the three functions, the Gaussian Estimator (GE) produces the lowest EER value, i.e. $8.55 \%$ when the input string is 4 digits long and 5.49\% when the input string is 16 digits long. This says that, even if the input string is known to the impersonator, 9 out of 10 impersonation attempts can be successfully identified. As the input string length increases, the success rate in identifying impersonation attempts also increases. These results are encouraging. They indicate the potential of using touch dynamics in conjunction with knowledge-based authentication to strengthen the security level of user authentication in mobile devices or service accesses via mobile devices. In addition, touch dynamics biometrics is cost-effective, as it does not require the use of additional hardware, and usable, as it is already part of the mobile device interface. So, it can be an attractive building block for other authentication solutions not only in a physical, but also a virtual environment. 


\subsubsection{With and Without Touch Dynamics}

One potential application area of touch dynamics biometrics is to integrate it into an existing authentication system to extend the system into a so-called multi-factor authentication system. Assuming a two-factor authentication system is used: one factor is PIN-based authentication and the other is touch dynamics authentication. Then an impersonator, to successfully sneak through the authentication verification process, would have to produce an acceptable touch pattern, in addition to successfully pass the PIN verification process. As shown in Table 5, in the case where the PIN is exposed, the chances for the impersonator to be successfully authenticated is drastically reduced from $100 \%$ (if only a PIN is used) to $12.21 \%$ (if both a 4-digit PIN and the touch dynamics are used) or to $9.43 \%$ (if both a 16 -digit PIN and the touch dynamics are used). To put things into perspective, assuming that there are 10 impersonation attempts, the system will only fail to detect once. Conversely, given the same case, the PIN-based authentication will fail to detect any of the impersonation attempts. However, the price to pay for this enhanced impersonation detection capability (i.e. enhanced security level) is that by using the touch dynamics based authentication method there is an increase of $4.89 \%$ (4-digit) or a $1.56 \%$ (16-digit) chance to reject a legitimate subject incorrectly. In other words, 1 out of 25 legitimate login attempts may be incorrectly rejected. With the PIN-based authentication method, on the other hand, a login attempt will not be rejected as long as the PIN entered is correct. In summary, with the use of the touch dynamics based multi-factor authentication method, there is a trade-off between security and usability. Our future effort is to investigate how to enhance or maintain the security level of this method, while minimising the usability cost.

\section{Table 5. The comparison between FAR and FRR with the presence of touch dynamics}

\section{RELATED WORK}

In this section, we provide a literature review of the related works with regard to evaluating the accuracy performance of touch dynamics biometrics and touch dynamics biometric data collections. We also highlight open issues and opportunities in the topic area.

\subsection{Performance Investigation}

Our related work review only focuses on touch dynamics biometrics using PIN-based input strings. The experimental work reported in paper (Sen and Muralidharan, 2014) was carried out to test the viability of identifying subjects based on touch dynamics biometrics using numerical input strings. In this experiment, only 10 subjects were recruited and each subject was asked to input a predefine PIN ("1593") on a HTC Nexus-One smartphone. To investigate if an impersonator could imitate another subject's touch pattern in the event if the subject's PIN is known to the impersonator, the author designed a visualisation tool to facilitate a separate set of attackers to imitate a legitimate subject's input pattern. Even by deliberately exposing the PIN, the timing and pressure feature information via the visualisation tool to the attackers, the method was still able to achieve a FAR value of $16 \%$. Though some interesting results were obtained from this experiment, the number of subjects used was too small to draw any conclusive remark. This is also the case for the work conducted by several other works (Amin et al., 2015; Buriro et al., 2015; Li et al., 2015).

The accuracy performance of touch dynamics applied on 4-digit PIN was also investigated by the authors in (de Mendizabal-Vazquez et al., 2014). They extracted data with regard to timing, finger touch size and pressure by using device build-in touchscreen sensors, and in addition, they also extracted linear and angular acceleration as feature vectors using accelerometers and gyroscopes sensors. As the size of data collected from these two sensors is large, they applied a pre-processing technique to reduce the size of the data. As a result, the computational resource required for data classification is reduced. However, the dataset in this experiment was collected in a quite constrained setting, where subjects had to hold the mobile phone in a fixed position. By using a Euclidean distances based classifier, they obtained a performance of $20 \%$ EER on a 4-digit PIN input. The performance comparisons of individual features were not given. Also, the use of the additional sensors (i.e. accelerometers and gyroscopes) to extract linear and angular acceleration feature data means that this technique may not be applicable to the mobile devices that are not equipped with these sensors.

(Zheng et al., 2014) also conducted their experiment on several 4- and 8-digit PINs. The authors employed a statistical one-class learning classifier and obtained average EER values of 3.65\%, 6.96\% and 7.34\% using three different 4-digit PIN numbers, "3244", " 1111 " and "5555", respectively. This shows that a higher repetition of digits can reduce accuracy performance. The experiment also compared the accuracy performances of two different 8-digit PINs (i.e. "12597384" and "12598416"). Surprisingly, the EER value of one of the 8-digit PIN ("12598416") was 4.45\%, marginally worse than the 4-digit PIN (“3244”). This contradicts with our experimental observation that a longer input string can produce a better accuracy performance. However, similar to our experimental observation, the accuracy performance provided by a combination of multiple feature data types outperforms that provided by a single feature data type.

The experiment reported in (Tasia et al., 2014) was carried out on a larger number of subjects than the experiments described above, and it used input PINs ranging from 4 to 8 digits long. An EER of $8.4 \%$ was achieved by using a simple statistical classifier. In this paper, the authors have also studied the time taken to perform the classification and verification of different PIN lengths and feature combinations; both consumed an average of $12 \mathrm{~ms}$ each. This experimental result is useful when considering the deployment of touch dynamics biometrics on power limited mobile devices.

The work reported in (Chang et al., 2015) was rather unique. The author proposed a method to allow subjects to change their PINs without rebuilding the classification model. The subjects were asked to input 10 different randomly selected 10-digits PINs. To reduce work burdens on the subjects, each subject was only required to provide 5 samples for each PIN. Based on all the samples collected, the authors produced a table of all possible feature data type values for each digit. They achieved EER values of $23 \%, 21 \%$ and $18 \%$ on three different PINs with the string lengths of 6, 8 and 10, respectively. This set of results is consistent with our experimental observation that, when the 16-digit PIN is used, a lower EER value can be achieved than using a 4-digit PIN. However, as the subjects involved in this experiment were at the age 17-20 years old, it is not clear whether the experimental results are applicable to other age groups.

Most of the experiments reported in literature extracted timing data from the two shortest possible feature lengths (i.e. uni-graph and digraph). An exception of these is the work carried out by (Trojahn et al., 2013). In this work, the authors compared the accuracy 


\begin{abstract}
performances of timing features produced from three different feature lengths (i.e. the uni-graph, di-graph and tri-graph) of a 17-digit PIN. The experimental result suggested that the uni-graph produces a better accuracy performance than the tri-graph. This may be due to the fact that the timing feature data expressed by a shorter feature length contains a higher level of granularity than a longer feature length. The authors also remarked that, by combining the results from uni-graph and di-graph, they could achieve a lower error rate. This is similar to the observations we made in our experiment that combining multiple feature data types produces a better accuracy performance than using a single feature data type. The similar observation has also been reported in other works such as (Tasia et al., 2014; Zheng et al., 2014).
\end{abstract}

By far, the best accuracy performance reported in literature was achieved by (Wu and Chen, 2015). They achieved an average EER value of $0.56 \%$. The work trained a classification model by using the Support Vector Machine (SVM) and both legitimate and illegitimate subject samples were used. In the experiment, each subject provided four different 8-digits PINs. Two main discoveries were made in their experiments. Firstly, a PIN with more repetitiveness (e.g. "11111111") resulted in a lower accuracy performance than a PIN with less repetitiveness (e.g. "16843752"). Secondly, the accuracy performance can be increased by increasing the size of legitimate subject samples, by using a combination of different feature data types and by pre-processing feature data in terms of data normalisation and outlier removal. In their experiment, they imposed a condition that the experimental subjects were selected from those that were very familiar with touchscreen smartphones. This condition might have played a role in achieving the high accuracy performance. It is not clear if the same level of accuracy performance could still be achieved if this condition is removed. Also, in their experiment, a two-class classifier was used to build the classification model. In other words, to build the classification model, samples from both legitimate and illegitimate subjects are required. However, in real-life, as mobile devices are very much personal devices, illegitimate subject samples may not be always available.

There were also experiments (Dhage et al., 2015; Giuffrida et al., 2014; Huang et al., 2012; Kambourakis et al., 2014) carried out on character-based passwords. As the scope of our work is on numerical PIN inputs, we do not discuss character-based experiments any further. A summary of the comparisons between our work and the related works is given in Table 6.

Table 6. Comparison to existing touch dynamics work carried out on PIN input

\title{
7.2 Public Datasets
}

As touchscreen devices only came along not long ago, there are still limited benchmark datasets publically available; so far, we are only able to find three such datasets, but only one of the datasets uses PIN based input and none of these is collected on a widescreen digital tablet.

The data collection process conducted by (El-Abed et al., 2014) involved 51 subjects. The input is a fixed password "rhu.university" entered on a virtual keyboard of a window touchscreen phone (Nokia Lumia 920). Each subject attended three sessions with an average of 5 days apart. The first of the three sessions was used as a practice session, so the actual data collection started from the second session. A total of 15 samples were collected from each subject in the two sessions. Only the timing feature was captured from this dataset, whereas our dataset also captures the finger touch size and pressure feature.

Another related effort on collecting and sharing their datasets publically was made by (Antal and Szabó, 2014). This work differs from ours in a number of ways. Firstly, the number of subjects involved is more than 3 times smaller than ours. Also the entire subject population recruited in this work was students, which is different from our case where the population consists of the members of a university as well as general public. In addition, different from our case where all the data were collected via the use of a single device, the data collection in this work was done via the use of two types of devices. 37 subjects provided their inputs on a Nexus 7 device, while the remaining 5 via the use of a LG Optimus L7 P700 smartphone. The paper did not explain if the use of the two device types would have any performance implications. To allow the sample data be used in EER estimations, the input string was predefined (".tie5Roanl"), which is also the case in our data collection. Also, in this work, the touch events captured include not only the input string but also shift key (toggle between lower and uppercase characters) and keyboard switch key (toggle between characters and numerical keys). These secondary key events may capture valuable and distinctive information about a subject. Inspired by this idea, in addition to capturing touch events on digit input, we have also recorded the Enter key event (pressed upon the completion of a PIN input) in our dataset. Also in this related work, most of the subjects provided their passwords for 30 times each on 2 isolated sessions in a period of two weeks (the time duration between the two sessions is not mentioned in the paper). As some invalid inputs were removed, so the resulting dataset only contain 51 input samples per subject (instead of 60 from both sessions).

(Tasia et al., 2014) has also reported a collection of a dataset based on numerical inputs. In this data collection process, the device used was an early generation smartphone with a physical keypad running on Android 2.0.1 (Éclair) API level 6, which was released in December 2009. By contrast, we adopted a more recent high resolution digital tablet with a later version of a mobile operating system. The subjects were only required to provide 2 samples per session and 5 sessions were used with an interval of at least 1 week apart for each session to eliminate intra-session typing variations. Different from our case where data were collected in a non-restrictive environment, their data were collected in a classroom, a rather confined environment. The age distribution of the subjects involved is biased towards young people, where $\mathrm{s} 85 \%$ of the total subjects has the age of 25 or younger. This is different from our case, where our dataset were collected from a diversified population and with different age groups and backgrounds. Also in this dataset, subjects were allowed to freely choose a PIN, and most of the chosen PINs have a length of 4 to 8 digits long. However, the actual PIN selections by each subject were not recorded in the shared dataset. In this dataset, raw finger touch sizes and pressure data have been recorded, and the timing feature was only recorded in a post-processed format (the duration and the latency). In other words, raw timing values were not recorded. This missing information may hinder the usability of the dataset in a wider context. Finally, different from the usual practice, test samples collected for the FAR test were collected separately from those for FRR test. 10 subjects are randomly chosen to act as impersonators. These impersonators were given the PINs of every other subject and were asked to impersonate the subjects by providing 5 samples for each subject. In this way, the samples 
used for the FRR tests cannot be reused for impersonation test samples, thus significantly reducing the size of the samples available for the FAR tests.

The creation and collection of live data is a time and resource consuming process, and this may be the reason for the lack of open datasets (Giot et al., 2009). However, the research, design and performance evaluation of touch dynamic biometrics systems require the availability of such benchmark datasets. To overcome these restrictions, we have collected touch dynamics data from 150 subjects. The dataset is made available to download at https://goo.gl/sNACU8.

An overview of presently available public datasets is summarised in Table 7, where $\mathrm{T}, \mathrm{S}$ and $\mathrm{P}$ indicate timing, finger touch size and pressure values, respectively.

\section{Table 7. Comparison of public datasets}

\subsection{Open Issues and Opportunities}

This section outlines open issues and potential research opportunities we have identified in doing our research.

Optimal Input Length. In general, the longer the input length, the better the accuracy performance we may achieve. However, the use of a longer input length increases the difficulty of remembering it by a subject, thus reducing usability. Further research work is necessary to study the trade-off between the accuracy performance and the usability.

Feature Data Enrichment. Exiting efforts on increasing the accuracy performance of touch dynamics biometrics have largely been focusing on using different classification techniques. An alternative way to increase the accuracy performance is by increasing the quality or variety of feature data. This can be done by deriving different types of feature from raw sensor data.

Effective Classification Technique. As the primary input for a classification technique in this problem context is subjects' touch pattern samples, we may also categorise samples based on the types of the samples used and determine the type of classifier that we should use. For example, a one-class classifier (e.g. distance measure) is modelled or trained by only using legitimate samples, and, a two-class classifier (e.g. neural network) is modelled or trained by using both legitimate and illegitimate samples. A mobile device is a highly personal device (rarely shared between multiple users), obtaining illegitimate samples in practice is not easy. So in the mobile device context, the use of a one-class classifier may be a more viable option. Further research work is necessary to study the accuracy performance difference between a one-class and two-class classifier.

Adaptive Learning. Human touch patterns are subject to change over time. To accommodate this change, a pattern adaptation method should be implemented in the underlying authentication system to update existing reference templates or classifier to reflect the most recent touch pattern of a subject. More work is necessary to evaluate the effectiveness of the adaptation approach.

Standardise Evaluation Criteria. There is an inconsistency in the performance evaluation metrics (as shown in Table 6) used by different researchers, and this has made it difficult to compare the accuracy performances produced using different methodologies and/or by different researchers. To facilitate effective comparisons of the related works, all the three standard accuracy performance evaluation metrics (i.e. FAR, FRR and EER) should be used.

\section{CONCLUSION}

Touch dynamics based authentication may provide us with a number of benefits, such as it is an inherent feature of a majority of the mobile devices already in use and it is readily deployable as an additional authentication factor to strengthen e-authentication assurance levels. This paper has investigated the feasibility and benefits of adopting a touch dynamics based authentication method by integrating it with the PIN based authentication method. To evaluate the effectiveness of this integrated approach, a proper dataset is required. With this motivation, we have reported how a comprehensive dataset are collected. The dataset can also serve further research on various issues in this context, such as further investigation and comparison of different classification methods or the potential use of touch dynamics for authentication purposes. We have then illustrated the extraction of different feature data from the dataset and how the captured feature data could be used for authentication to identify a user. We have also applied three light-weight matching functions to the dataset to study their accuracy performances. The matching functions used in this experiment were chosen on the ground that they are computationally less expensive than the other functions, so the resulting authentication system could consumes less power and introduces less delay in authenticating a user. We have also investigated how the accuracy performance may be influenced by variations in factors such as the timing resolution of timing feature data, combinations of different feature data types, input string lengths and subject sizes. Experimental results show that, with the use of the two-factor authentication method, even if an impersonator knows the input string (i.e. PIN) of a legitimate subject, 9 out of 10 impersonation attempts can be successfully identified. We also showed that the accuracy performance can be increased by combining different feature data types. The results we have obtained so far demonstrate that touch dynamics biometrics can be an effective solution to strengthen the security level offered to mobile devices.

\section{REFERENCES}

Amin, R., Gaber, T. and ElTaweel, G. (2015), "Implicit Authentication System for Smartphones Users Based on Touch Data", in Abraham, A., Jiang, X.H., Snášel, V. and Pan, J.-S. (Eds.), Intelligent Data Analysis and Applications, Springer International Publishing, pp. 251-262.

Antal, M. and Szabó, L.Z. (2014), "Keystroke Dynamics on Android Platform”, Proceedings of the 8th International Conference Interdisciplinarity in Engineering, Romania, pp. 131-136.

Bleha, S., Slivinsky, C. and Hussien, B. (1990), "Computer-access security systems using keystroke dynamics", Pattern Analysis and Machine Intelligence, IEEE Transactions on, Vol. 12 No. 12, pp. 1217-1222. 
Bo, C., Zhang, L., Jung, T., Han, J., Li, X.-Y. and Wang, Y. (2014), “Continuous user identification via touch and movement behavioral biometrics", Performance Computing and Communications Conference (IPCCC), 2014 IEEE International, presented at the Performance Computing and Communications Conference (IPCCC), 2014 IEEE International, pp. 1-8.

Buriro, A., Crispo, B., Frari, F.D. and Wrona, K. (2015), "Touchstroke: Smartphone User Authentication Based on Touch-Typing Biometrics", in Murino, V., Puppo, E., Sona, D., Cristani, M. and Sansone, C. (Eds.), New Trends in Image Analysis and Processing -- ICIAP 2015 Workshops, Springer International Publishing, pp. 27-34.

Buschek, D., De Luca, A. and Alt, F. (2015), "Improving Accuracy, Applicability and Usability of Keystroke Biometrics on Mobile Touchscreen Devices", To Appear: Proceedings of the SIGCHI Conference on Human Factors in Computing Systems.

Campisi, P., Maiorana, E., Lo Bosco, M. and Neri, A. (2009), "User authentication using keystroke dynamics for cellular phones", Iet Signal Processing, Vol. 3 No. 4, pp. 333-341.

Chang, T.-Y., Tsai, C.-J., Tsai, W.-J., Peng, C.-C. and Wu, H.-S. (2015), “A changeable personal identification number-based keystroke dynamics authentication system on smart phones”, Security and Communication Networks, p. n/a-n/a.

Chong, M.K., Marsden, G. and Gellersen, H. (2010), "GesturePIN: Using Discrete Gestures for Associating Mobile Devices", Proceedings of the 12th International Conference on Human Computer Interaction with Mobile Devices and Services, ACM, New York, NY, USA, pp. 261-264.

Cho, S., Han, C., Han, D.H. and Kim, H.-I. (2000), "Web-Based Keystroke Dynamics Identity Verification Using Neural Network", Journal of Organizational Computing and Electronic Commerce, Vol. 10 No. 4, pp. 295-307.

Cisco. (2015), “Cisco Visual Networking Index: Global Mobile Data Traffic Forecast Update 2014-2019”, Cisco, 3 February, available at: http://www.cisco.com/en/US/solutions/collateral/ns341/ns525/ns537/ns705/ns827/white_paper_c11-520862.html (accessed 14 April 2012).

Clarke, N.L. and Furnell, S.M. (2007), “Authenticating mobile phone users using keystroke analysis", International Journal of Information Security, Vol. 6 No. 1, pp. 1-14.

Crawford, H., Renaud, K. and Storer, T. (2013), “A framework for continuous, transparent mobile device authentication”, Computers \& Security, Vol. 39, Part B, pp. 127-136.

De Luca, A., Hang, A., Brudy, F., Lindner, C. and Hussmann, H. (2012), “Touch Me Once and I Know It's You!: Implicit Authentication Based on Touch Screen Patterns", Proceedings of the SIGCHI Conference on Human Factors in Computing Systems, ACM, New York, NY, USA, pp. 987-996.

Dhage, S., Kundra, P., Kanchan, A. and Kap, P. (2015), "Mobile authentication using keystroke dynamics", 2015 International Conference on Communication, Information Computing Technology (ICCICT), presented at the 2015 International Conference on Communication, Information Computing Technology (ICCICT), pp. 1-5.

Draffin, B., Zhu, J. and Zhang, J. (2014), "KeySens: Passive User Authentication through Micro-behavior Modeling of Soft Keyboard Interaction", in Memmi, G. and Blanke, U. (Eds.), Mobile Computing, Applications, and Services, Springer International Publishing, pp. 184-201.

El-Abed, M., Dafer, M. and El Khayat, R. (2014), "RHU Keystroke: A mobile-based benchmark for keystroke dynamics systems", 2014 International Carnahan Conference on Security Technology (ICCST), presented at the 2014 International Carnahan Conference on Security Technology (ICCST), pp. 1-4.

Gaines, R.S., Lisowski, W., Press, S.J. and Shapiro, N. (1980), Authentication by Keystroke Timing: Some Preliminary Results, No. R2526-NSF, Rand Corporation, Santa Monica, CA.

Gascon, H., Uellenbeck, S., Wolf, C. and Rieck, K. (2014), "Continuous authentication on mobile devices by analysis of typing motion behavior", Lecture Notes in Informatics, Vol. P-228, pp. 1-12.

Giot, R., El-Abed, M. and Rosenberger, C. (2009), "GREYC keystroke: A benchmark for keystroke dynamics biometric systems", Biometrics: Theory, Applications, and Systems, 2009. BTAS '09. IEEE 3rd International Conference on, pp. 1-6.

Giuffrida, C., Majdanik, K., Conti, M. and Bos, H. (2014), "I Sensed It Was You: Authenticating Mobile Users with Sensor-Enhanced Keystroke Dynamics", in Dietrich, S. (Ed.), Detection of Intrusions and Malware, and Vulnerability Assessment, Springer International Publishing, pp. 92-111.

Huang, X., Lund, G. and Sapeluk, A. (2012), "Development of a Typing Behaviour Recognition Mechanism on Android", 2012 IEEE 11 th International Conference on Trust, Security and Privacy in Computing and Communications (TrustCom), presented at the 2012 IEEE 11th International Conference on Trust, Security and Privacy in Computing and Communications (TrustCom), pp. 13421347.

Kambourakis, G., Damopoulos, D., Papamartzivanos, D. and Pavlidakis, E. (2014), "Introducing touchstroke: keystroke-based authentication system for smartphones", Security and Communication Networks, available at:http://doi.org/10.1002/sec.1061.

Kim, D., Dunphy, P., Briggs, P., Hook, J., Nicholson, J.W., Nicholson, J. and Olivier, P. (2010), "Multi-touch authentication on tabletops", Proceedings of the 28th International Conference on Human Factors in Computing Systems, Vol. Atlanta, Georgia, USA, ACM, New York, NY, USA, pp. 1093-1102.

Kolly, S.M., Wattenhofer, R. and Welten, S. (2012), "A Personal Touch: Recognizing Users Based on Touch Screen Behavior", Proceedings of the Third International Workshop on Sensing Applications on Mobile Phones, ACM, New York, NY, USA, pp. 1:1-1:5.

Li, Y., Yang, J., Xie, M., Carlson, D., Jang, H.G. and Bian, J. (2015), “Comparison of PIN- and pattern-based behavioral biometric authentication on mobile devices", MILCOM 2015 - 2015 IEEE Military Communications Conference, presented at the MILCOM 2015 - 2015 IEEE Military Communications Conference, pp. 1317-1322.

Loy, C.C., Lai, W.K. and Lim, C.P. (2007), "Keystroke Patterns Classification Using the ARTMAP-FD Neural Network", Intelligent Information Hiding and Multimedia Signal Processing, 2007. IIHMSP 2007. Third International Conference on, Vol. 1, pp. 61 64. 
Maiorana, E., Campisi, P., González-Carballo, N. and Neri, A. (2011), "Keystroke dynamics authentication for mobile phones", Proceedings of the 2011 ACM Symposium on Applied Computing, ACM, New York, NY, USA, pp. 21-26.

de Mendizabal-Vazquez, I., de Santos-Sierra, D., Guerra-Casanova, J. and Sanchez-Avila, C. (2014), "Supervised classification methods applied to keystroke dynamics through mobile devices", 2014 International Carnahan Conference on Security Technology (ICCST), presented at the 2014 International Carnahan Conference on Security Technology (ICCST), pp. 1-6.

Ngugi, B., Kahn, B.K. and Tremaine, M. (2011), "Typing Biometrics: Impact of Human Learning on Performance Quality", J.Data and Information Quality, Vol. 2 No. 2, pp. 11:1-11:21.

Ngugi, B., Tremaine, M. and Tarasewich, P. (2011), "Biometric keypads: Improving accuracy through optimal PIN selection.", Decision Support Systems, Vol. 50 No. 4, pp. 769-776.

Niu, Y. and Chen, H. (2012), "Gesture Authentication with Touch Input for Mobile Devices", in Prasad, R., Farkas, K., Schmidt, A.U., Lioy, A., Russello, G. and Luccio, F.L. (Eds.), Security and Privacy in Mobile Information and Communication Systems, Springer Berlin Heidelberg, pp. 13-24.

Obaidat, M.S. (1995), “A verification methodology for computer systems users", Proceedings of the 1995 ACM Symposium on Applied Computing, Vol. Nashville, Tennessee, United States, ACM, pp. 258-262.

Robinson, J.A., Liang, V.M., Chambers, J.A.M. and MacKenzie, C.L. (1998), "Computer user verification using login string keystroke dynamics", Ieee Transactions on Systems Man and Cybernetics Part a-Systems and Humans, Vol. 28 No. 2, pp. $236-241$.

Saravanan, P., Clarke, S., Chau, D.H. (Polo) and Zha, H. (2014), "LatentGesture: Active User Authentication Through Background Touch Analysis", Proceedings of the Second International Symposium of Chinese CHI, ACM, New York, NY, USA, pp. 110-113.

Sen, S. and Muralidharan, K. (2014), "Putting 'pressure' on mobile authentication", 2014 Seventh International Conference on Mobile Computing and Ubiquitous Networking (ICMU), presented at the 2014 Seventh International Conference on Mobile Computing and Ubiquitous Networking (ICMU), pp. 56-61.

Serwadda, A., Phoha, V.V. and Wang, Z. (2013), "Which verifiers work?: A benchmark evaluation of touch-based authentication algorithms", 2013 IEEE Sixth International Conference on Biometrics: Theory, Applications and Systems (BTAS), presented at the 2013 IEEE Sixth International Conference on Biometrics: Theory, Applications and Systems (BTAS), pp. 1-8.

Stewart, J.C., Monaco, J.V., Cha, S.-H. and Tappert, C.C. (2011), "An investigation of keystroke and stylometry traits for authenticating online test takers", Biometrics (IJCB), 2011 International Joint Conference on, pp. 1-7.

Tasia, C.-J., Chang, T.-Y., Cheng, P.-C. and Lin, J.-H. (2014), "Two novel biometric features in keystroke dynamics authentication systems for touch screen devices", Security and Communication Networks, Vol. 7 No. 4, pp. 750-758.

Teh, P.S., Teoh, A.B.J. and Yue, S. (2013), “A Survey of Keystroke Dynamics Biometrics”, The Scientific World Journal, Vol. 2013, p. e408280.

Trojahn, M., Arndt, F. and Ortmeier, F. (2013), “Authentication with Keystroke Dynamics on Touchscreen Keypads - Effect of different N-Graph Combinations", presented at the MOBILITY 2013, The Third International Conference on Mobile Services, Resources, and Users, pp. 114-119.

Wu, J. and Chen, Z. (2015), "An Implicit Identity Authentication System Considering Changes of Gesture Based on Keystroke Behaviors", International Journal of Distributed Sensor Networks, Vol. 2015, p. e470274.

Xu, H., Zhou, Y. and Lyu, M.R. (2014), "Towards Continuous and Passive Authentication via Touch Biometrics: An Experimental Study on Smartphones", Symposium On Usable Privacy and Security (SOUPS 2014), USENIX Association, Menlo Park, CA, pp. 187198.

Zheng, N., Bai, K., Huang, H. and Wang, H. (2014), "You Are How You Touch: User Verification on Smartphones via Tapping Behaviors", 2014 IEEE 22nd International Conference on Network Protocols (ICNP), presented at the 2014 IEEE 22 nd International Conference on Network Protocols (ICNP), pp. 221-232. 
Table 1. Subject demography of our dataset

\begin{tabular}{|c|c|c|c|c|}
\hline \multicolumn{2}{|c|}{ Properties } & \multicolumn{3}{c|}{ Details } \\
\hline \multicolumn{2}{|c|}{ Subjects } & 50 & 100 & 150 \\
\hline \multirow{2}{*}{ Population } & Academia & 9 & 11 & 18 \\
\cline { 2 - 5 } & Public & 41 & 88 & 132 \\
\hline \multirow{3}{*}{ Age } & $<20$ & 8 & 13 & 28 \\
\cline { 2 - 5 } & $20-40$ & 27 & 49 & 66 \\
\cline { 2 - 5 } & $>40$ & 15 & 38 & 56 \\
\hline \multirow{3}{*}{ Gender } & Male & 12 & 24 & 45 \\
\cline { 2 - 5 } & Female & 38 & 76 & 105 \\
\hline \multirow{2}{*}{ Usage Frequency } & Rare & 16 & 32 & 49 \\
\cline { 2 - 5 } & Average & 12 & 20 & 32 \\
\cline { 2 - 5 } & Often & 22 & 48 & 69 \\
\hline \multirow{2}{*}{ Hand Preference } & Left-hand & 5 & 8 & 14 \\
\cline { 2 - 5 } & Right-hand & 45 & 92 & 136 \\
\hline
\end{tabular}

Table 2. EER values of different feature data type combinations

\begin{tabular}{|c|c|c|c|}
\hline \multirow{2}{*}{ Feature Data Types } & \multirow{2}{*}{$\begin{array}{c}\text { No. of } \\
\text { Feature }\end{array}$} & 4-Digit & 16-Digit \\
\cline { 3 - 4 } & 1 & 15.98 & 12.44 \\
\hline$P S$ & 2 & $10.17(+36.36 \%)$ & $8.12(+49.19 \%)$ \\
\hline$F T_{4}, P S$ & 3 & $7.63(+52.25 \%)$ & $7.67(+52.00 \%)$ \\
\hline$D T, F T_{1}, P S$ & 4 & $7.48(+53.19 \%)$ & $6.39(+60.01 \%)$ \\
\hline$D T, F T_{1}, F T_{4}, P S$ & 5 & $7.75(+51.50 \%)$ & $6.33(+60.39 \%)$ \\
\hline$D T, F T_{1}, F T_{2}, F T_{4}, P S$ & 6 & $7.71(+51.75 \%)$ & $\mathbf{6 . 2 7}(+60.76 \%)$ \\
\hline$D T, F T_{1}, F T_{2}, F T_{3}, F T_{4}, P S$ & \multicolumn{3}{|c}{} \\
\hline
\end{tabular}

Table 3. EER values vs subject sizes

\begin{tabular}{|c|c|c|c|c|}
\hline \multirow{2}{*}{ Classifier } & \multicolumn{2}{|c|}{ 4-Digit } & \multicolumn{2}{c|}{ 16-Digit } \\
\cline { 2 - 5 } & 50 Subjects & 150 Subjects & 50 Subjects & 150 Subjects \\
\hline GE & 7.71 & 8.55 & 6.27 & 5.49 \\
\hline ZS & 8.51 & 9.30 & 6.70 & 5.54 \\
\hline SD & 7.57 & 8.92 & 7.45 & 5.74 \\
\hline Average EER & 7.93 & 8.92 & 6.81 & 5.59 \\
\hline
\end{tabular}

Table 4. Performance between classifiers on different input lengths

\begin{tabular}{|c|c|c|c|c|c|c|}
\hline \multirow{2}{*}{ Classifier } & \multicolumn{3}{|c|}{ 4 Digits } & \multicolumn{3}{c|}{ 16 Digits } \\
\cline { 2 - 7 } & FAR & FRR & EER & FAR & FRR & EER \\
\hline GE & 12.21 & 4.89 & $\mathbf{8 . 5 5}$ & 9.43 & 1.56 & $\mathbf{5 . 4 9}$ \\
\hline ZS & 15.27 & 3.33 & 9.30 & 8.64 & 2.44 & 5.54 \\
\hline SD & 8.95 & 8.89 & 8.92 & 10.36 & 1.11 & 5.74 \\
\hline
\end{tabular}

Table 5. The comparison between FAR and FRR with the presence of touch dynamics

\begin{tabular}{|c|c|c|}
\hline Feature & FAR & FRR \\
\hline PIN & 100 & 0 \\
\hline PIN (4-digits) + Touch Dynamics & 12.21 & 4.89 \\
\hline PIN (16-digits) + Touch Dynamics & 9.43 & 1.56 \\
\hline
\end{tabular}


Table 6. Comparison to existing touch dynamics work carried out on PIN input

\begin{tabular}{|c|c|c|c|c|c|c|c|}
\hline Paper & Length & Subjects & Input & Device & FAR & FRR & EER \\
\hline (Trojahn et al., 2013) & 17 & 152 & Fixed & Samsung Galaxy Nexus & 4.19 & 4.59 & - \\
\hline (Sen and Muralidharan, 2014) & 4 & 10 & Fixed & HTC Nexus-One & 14.1 & 14.1 & 15.2 \\
\hline \multirow{2}{*}{ (Zheng et al., 2014) } & 4 & \multirow{2}{*}{80} & \multirow{2}{*}{ Fixed } & \multirow{2}{*}{ Samsung Galaxy Nexus } & - & - & 3.65 \\
\hline & 8 & & & & - & - & 4.45 \\
\hline (de Mendizabal-Vazquez et al., 2014) & 4 & 80 & Fixed & - & - & - & 20 \\
\hline (Tasia et al., 2014) & $4-10$ & 100 & Random & Motorola Milestone & 8.4 & 8.32 & 8.4 \\
\hline (Wu and Chen, 2015) & 8 & 100 & Fixed & - & - & - & 0.56 \\
\hline (Buriro et al., 2015) & 4 & 12 & Fixed & Google Nexus 5 & 1 & 1 & - \\
\hline (Li et al., 2015) & 4 & 15 & Fixed & HTC Droid DNA & - & - & 4.2 \\
\hline (Amin et al., 2015) & 7 & 12 & Fixed & Samsung Galaxy Note N7000 & 13.9 & 0.53 & - \\
\hline \multirow{3}{*}{ (Chang et al., 2015) } & 6 & \multirow{3}{*}{100} & \multirow{3}{*}{ Random } & \multirow{3}{*}{ HTC Desire Z } & - & - & 23 \\
\hline & 8 & & & & - & - & 21 \\
\hline & 10 & & & & - & - & 18 \\
\hline \multirow{2}{*}{ This Paper } & 4 & \multirow{2}{*}{150} & \multirow{2}{*}{ Fixed } & \multirow{2}{*}{ Samsung Galaxy Tab 10.1} & 12.21 & 4.89 & 8.55 \\
\hline & 16 & & & & 9.43 & 1.56 & 5.49 \\
\hline
\end{tabular}

Table 7. Comparison of public datasets

\begin{tabular}{|c|c|c|c|c|c|c|c|}
\hline Dataset & Subject & Population & Sample & Input & Feature & Setting & Platform \\
\hline (El-Abed et al., 2014) & 51 & Restricted & 15 & "rhu.university" & $\mathrm{T}$ & Confined & Phone \\
\hline (Antal and Szabó, 2014) & 42 & Restricted & 51 & “.tie5Roanl” & $\mathrm{T}, \mathrm{S}, \mathrm{P}$ & Confined & Phone \\
\hline (Tasia et al., 2014) & 100 & Restricted & 5 & 6 to 8 digits & T,S,P & Confined & Phone \\
\hline This Paper & 150 & Diversified & 10 & “5560”, “1379666624680852” & $\mathrm{T}, \mathrm{S}, \mathrm{P}$ & Flexible & Tablet \\
\hline
\end{tabular}

